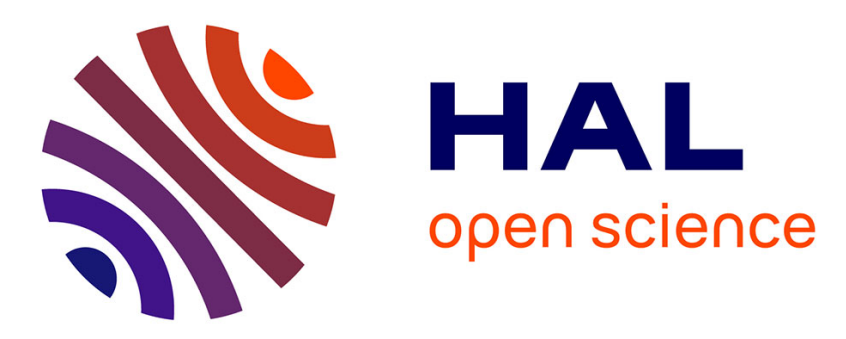

\title{
Quantifying the Achievable Cellular Traffic Offloading Gain with Passpoint Hotspots
}

Sahar Hoteit, Stefano Secci, Guy Pujolle, Sven Wietholter, Adam Wolisz, Cezary Ziemlicki, Zbigniew Smoreda

\section{To cite this version:}

Sahar Hoteit, Stefano Secci, Guy Pujolle, Sven Wietholter, Adam Wolisz, et al.. Quantifying the Achievable Cellular Traffic Offloading Gain with Passpoint Hotspots. WiMobCity'14 - ACM international workshop on Wireless and mobile technologies for smart cities, Aug 2014, Philadelphia, United States. pp.19-28, 10.1145/2633661.2633672 . hal-01131518

\section{HAL Id: hal-01131518 https://hal.science/hal-01131518}

Submitted on 20 Mar 2015

HAL is a multi-disciplinary open access archive for the deposit and dissemination of scientific research documents, whether they are published or not. The documents may come from teaching and research institutions in France or abroad, or from public or private research centers.
L'archive ouverte pluridisciplinaire HAL, est destinée au dépôt et à la diffusion de documents scientifiques de niveau recherche, publiés ou non, émanant des établissements d'enseignement et de recherche français ou étrangers, des laboratoires publics ou privés. 


\section{Quantifying the Achievable Cellular Traffic Offloading Gain with Passpoint Hotspots}

\author{
Sahar Hoteit, \\ Stefano Secci, \\ Guy Pujolle \\ LIP6, UPMC \\ 4 place Jussieu, 75005 Paris, \\ France \\ firstname.lastname@lip6.fr
}

\author{
Sven Wietholter, \\ Adam Wolisz \\ Technical University Berlin \\ Einsteinufer 25, 10587 Berlin, \\ Germany \\ lastname@tkn.tu-berlin.de
}

\author{
Cezary Ziemlicki, \\ Zbigniew Smoreda \\ SENSe, Orange labs \\ 38 rue du G. Leclerc, 92794 \\ Issy-les-Moulineaux, France \\ firstname.lastname@orange.com
}

\begin{abstract}
Wi-Fi technology has always been an attractive solution for catering the increasing data demand in mobile networks because of the availability of Wi-Fi networks, the high bit rates they provide, and the lower cost of ownership. However, the legacy WiFi technology lacks of seamless interworking between Wi-Fi and mobile cellular networks on the one hand, and between Wi-Fi hotspots on the other hand. Nowadays, the recently released Wi-Fi Certified Passpoint Program provides the necessary control-plane for these operations. Service providers can henceforth look to such Wi-Fi systems as a viable way to seamlessly offload mobile traffic and deliver addedvalue services, so that subscribers no longer face the frustration and aggravation of connecting to Wi-Fi hotspots. However, the technology being rather recent, we are not aware of public studies at the state of the art documenting the achievable gain in real mobile networks. In this paper, we evaluate the capacity gain that one can get by offloading cellular data traffic over Wi-Fi Passpoint hotspots as a function of different hotspot placement schemes and of access point selection policies (two enabled by the Passpoint control-plane and one independent of it). We compare the policies using real mobile data from the Orange network in Paris. We show that offloading using Passpoint control-plane information can grant up to $15 \%$ gain with respect to Passpoint-agnostic ones based on signal quality information. As of placement strategy, installing Passpoint hotspots in the outer annulus of the macrocell coverage grants the maximum gains.
\end{abstract}

\section{Keywords}

Mobile Data Traffic; Passpoint; Traffic Offloading

\section{INTRODUCTION}

Mobile data traffic continues its tremendous growth path, with an increasing number of smartphones, tablets and high-end handsets requiring ubiquitous Internet access. As a side effect of this mobile data explosion, we face nowadays the challenge of managing traffic overloads in cellular networks. According to the techni-

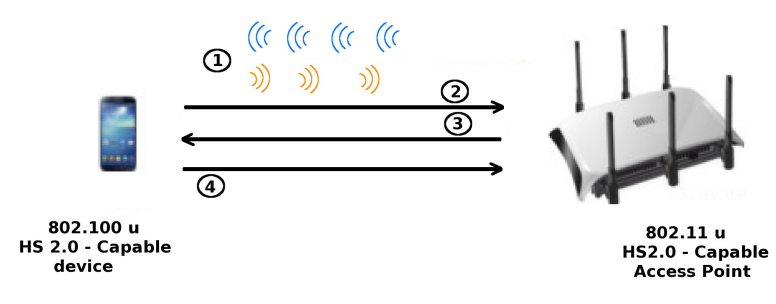

Figure 1: Passpoint hotspot association.

cal report [1], mobile data traffic will grow at a compound annual growth rate of $66 \%$ from 2012 to 2017, reaching 11.2 Exabytes per month by 2017. In order to meet mobile Internet demand while addressing the lack of available mobile spectrum and the expense of new infrastructure, service providers are severely challenged. They need to master the needed capacity expansion in their backhauling network, otherwise the data traffic will sooner or later clog their networks. Next-generation network deployments promise to deliver higher bandwidth and speed, but they often imply high capital and operational expenditures [2].

An alternative economically and technically viable way is represented by mobile data offloading solutions. Such solutions aim to optimize the resource utilization reducing the traffic on operator's licensed spectrum, and lowering the traffic load on base stations. Wi-Fi technology has always been an attractive solution for data offloading because of the ubiquity of Wi-Fi networks, the high bit rates they provide, the simplicity in deployment and maintenance, and the lower CAPEX [3]. Until the Wi-Fi Certified Passpoint Program (also known as 'Hotspot 2.0' and referred to in the following shortly as 'Passpoint') [4], the WiFi technology was lacking of seamless interworking between Wi-Fi and mobile cellular networks on the one hand and between Wi-Fi hotspots on the other hand. The new Passpoint program aims to make the WiFi network a "true extension of service provider networks", letting users roam from one hotspot to another with no manual effort, just like cell phone network that already switches seamlessly from one cell tower to another. The Passpoint technology provides all controlplane functionalities for automated and seamless connectivity to Wi-Fi hotspots. With Passpoint, service providers can look to such advanced Wi-Fi systems as a viable way to offload traffic and deliver high-bandwidth services. At the same time, subscribers no longer have to face the frustration and service degradation typically experienced when connecting to legacy Wi-Fi hotspots.

As a matter of fact, Passpoint can work in any network and over- 
Table 1: Beacon and probe response information elements in Passpoint.

\begin{tabular}{c||c}
\hline Access Network Type & identifies whether hotspot is for public, private or guest access. \\
\hline Internet Bit & indicates if the hotspot can be used for Internet access. \\
\hline Advertisement Protocol & indicates the hotspot supports GAS/ANQP \\
\hline Roaming Consortium element & provide a list of up to 3 names of reachable service providers. \\
\hline Venue Information & describes the venue where the hotspot is situated. \\
\hline Load Element & provides information on channel utilization and the current number of associated devices. \\
\hline
\end{tabular}

comes the limitations of proprietary, non-interoperable solutions offered by some providers today. Devices certified in the Passpoint program will be able to manage network association, authentication, sign-up, and security in the background, in a way that is completely transparent to the subscriber and that consistently works in any Passpoint network [5] [6]. When a user with a "Hotspot 2.0" (HS2.0) capable mobile device (i.e., based on IEEE 802.11u) comes within the range of a HS2.0 capable hotspot, it will automatically open up a dialog with that hotspot to determine its capabilities before proceeding to authentication. It is worth noting that Passpoint logic is already implemented in many mobile devices, such as Android-based ones. Moreover, since Passpoint discovery is based on pre-authentication, there are considerable savings of time and battery life compared to existing methods [4].

In this paper, we evaluate mobile data traffic offloading over Passpoint hotspots by determining the obtainable capacity gain in dense urban environment. For the assessment, we use real Orange cellular network dataset retrieved by probes capturing mobile data sessions' details, and we compare different hotspot selection policies enabled by Passpoint with each other and with a Passpoint-agnostic policy based on signal quality metrics. Basic Passpoint policies can be based on the least utilized channel or the least number of attached users. The Passpoint-agnostic policy is one selecting the hotspot with the highest signal to noise ratio. We find out that offloading using Passpoint control-plane information can grant up to $15 \%$ gain with respect to Passpoint-agnostic ones based on signal quality information. Moreover, we show that installing Passpoint hotspots in the outer annulus of the macrocell coverage permits to increase the offloading system capacity and system performance. The paper is organized as follows. Section 2 presents Passpoint and gives an overview of related works. Section 3 synthetically presents the available dataset and reports data traffic consumption and users characteristics. Section 4 describes the offloading over Passpoint approach, followed by a presentation of simulation results in Section 5. Finally, Section 6 concludes the paper.

\section{BACKGROUND}

In the following, we first give an insight on the hotspot-device signaling information exchanged with Passpoint, and then we provide an overview of relevant work on the matter at the state of the art.

\subsection{Passpoint Hotspot-Device Signaling}

Fig. 1 illustrates the four different required steps for Passpoint hotspot association. The Access Network Query Protocol (ANQP) is used for device-hotspot signaling [4].

1. The $802.11 \mathrm{u}$-capable access point broadcasts its HS2.0 support, so that HS2.0-enabled devices can recognise such support.

2. The $802.11 \mathrm{u}$-capable device is able to process ANQP messages, containing useful information such as the 'reachable' authenticators, and various hotspot capabilities. The $802.11 \mathrm{u}-$ capable device requests full authenticators list.

3. The hotspot responds to the ANQP query with the requested information.

4. Device compares provisioned network-selection policy with HS2.0 data from hotspots and associates itself to the best hotspot suitable for its needs.

Table 1 shows some of the information elements provided by the hotspot to the mobile devices. In the specifications, those six elements are mentioned. Most elements provide simple configuration and network reachability and locality information. The most interesting element for efficient Passpoint selection is the Load Element, which allows a mobile device to be informed about hotspot channel utilization and the current number of associated devices to a Passpoint hotspot.

\subsection{Related works}

The increasing need of offloading solutions is caused by the explosion of Internet data traffic, especially the growing portion of traffic going through mobile networks. For these reasons, different studies and researches tackling mobile data offloading have been conducted in the past few years to alleviate the traffic load on cellular networks. We present in the following some of the offloading approaches proposed thus far. Wi-Fi and femtocell technologies are considered the primary offload technologies considered today by the industry stakeholders.

\subsubsection{Horizontal data offloading}

The femtocell technology [7] [8], also referred to as small-cells technology, aims to offer better indoor voice and data services for cellular networks via the deployment of tiny cellular repeaters, differently backhauled and synchronized. Femtocell services are already commercialized to expand cell coverage and improve radio resource management [9]. Femtocells work on the same licensed spectrum as the macrocells of cellular networks and thus do not require special hardware support on mobile phones, thus simplifying data offloading procedures. But, despite the benefits of femtocells networks in offloading data traffic via horizontal handovers from macro to femto cells and vice versa, one should not forget the inherent constraints of such networks due to cross-tier and cotier interferences that should be taken into account when installing femtocells [10].

The cross-tier interference [11] is defined as the decrease in signal quality of macrocell users due to the presence of femto users sharing the same spectrum and vice versa, and the co-tier interference occurs when all femtocells share the same spectrum. Advanced resource scheduling and allocation techniques have been defined for both spectrum management situations, such as [12] for cross-tier and [13] [14] for co-tier interference. Despite the promising results therein in terms of achievable performance, those approaches either require a form of explicit coordination and signal- 
ing among femtocells or group of femtocells, or some sort of centralization to collect necessary multi-cell information at one computing place (e.g., using Cloud Radio Access Network, C-RAN, solutions [15]). In either case, an important level of complexity and significant investments need to be undertaken to implement this type of offloading management.

\subsubsection{Vertical data offloading}

A much simpler, inexpensive and lightweight solution consists of using Wi-Fi hotspots for data offloading. The key advantage of offloading to Wi-Fi hotspots is that they operate over unlicensed spectrum, thus no interference management is required between macrocell and Wi-Fi hotspots. In addition, the installation of WiFi hotspots is easier and more cost effective than large cellular network deployments and upgrades. The main problem that was facing the industry with Wi-Fi is that it is used only for fixed access. Nevertheless, nowadays with the Wi-Fi Certified Passpoint this problem is overcome; in other terms, this new standard enables seamless hopping from hotspot to hotspot and even vertical handoffs across cellular and Wi-Fi networks without the user being aware of it [4] [5].

Likely because of its recent specification, the scientific papers discussed from the literature do not consider the Passpoint technology explicitly along with its hotspot selection capabilities. We present thereafter a selection of Wi-Fi offloading strategies available in the literature.

Authors in [16] measure the offloading potential of the public WiFi based on city wide vehicular traces. Compared to the vehicle based high mobility scenario in [16], the authors in [17] study the performance of $3 \mathrm{G}$ mobile data offloading through Wi-Fi networks in a more general mobile scenario with empirical pedestrian traces. They distinguish two different types of Wi-Fi offloading: on-thespot and delayed offloading. The first type consists of spontaneous connectivity to Wi-Fi and transfer data on the spot; when users move outside the Wi-Fi coverage area, the offloading is stopped and all the unfinished transfers are transmitted back to cellular networks. In the delayed offloading, each data transfer is associated with a deadline and as users come in and out of Wi-Fi coverage areas, their data transfer is repeatedly resumed until the transfer is complete or the deadline is reached. Based on a study done over some smartphones users and on the statistical distributions of their Wi-Fi connectivity, the authors evaluate the Wi-Fi offloading efficiency for various amount of Wi-Fi deployment, different deployment strategies, different traffic intensity and delay deadlines, showing that Wi-Fi in such configurations can offload up to $65 \%$ of the total mobile data traffic. Similarly, the authors in [18] explore the benefits in terms of energy savings that can be achieved by offloading traffic loads to Wi-Fi networks. Using different traffic types, they show that a saving of up to $70 \%$ is reached by opportunistically powering down cellular radio network equipment to offload users traffic to Wi-Fi hotspots.

In [19], a WiFi offloading scheme is proposed from a transport layer perspective. A multipath protocol called oSCTP is proposed to offload the $3 \mathrm{G}$ traffic via WiFi networks and maximize the user's benefit. The philosophy of oSCTP is to use WiFi and 3G interfaces simultaneously if necessary, and schedule packets transmitted in each interface every schedule interval. By modeling user utility and cost both as a function of the $3 \mathrm{G}$ and WiFi network usage, the user's benefit, i.e., the difference between the utility and the cost, is maximized through an optimization problem. Following the same direction, the authors in [20] propose a framework for 3G traffic offloading based on the idea of motivating mobile users with high delay tolerance to offload their traffic to Wi-Fi networks. A feasible approach consists of delaying all delay tolerant applications until their maximum delay tolerance, and then resorting to the cellular networks if the applications can not finish. However, this approach does not appear much effective, considering that the user has to wait even when there is actually no available Wi-Fi connection. To solve this problem, the authors in [21] propose an adaptive approach that computes an offload handing-back time, after which the user stops waiting for offloading through Wi-Fi connections, hence resorting to the cellular network service. This allows achieving a better tradeoff between offloaded volume and user satisfaction.

\section{ON MOBILE DATA CHARACTERISTICS}

It is of paramount importance to have a realistic insight on real mobile data characteristics to understand the potential impact of offloading techniques at large. In this section, after a brief description of the available dataset, we synthetically describe mobile data consumption characteristics.

\subsection{Cellular Network Dataset}

The dataset used in our study consists of network probe's data, generated each time a mobile device uses the wireless mobile network for Internet data exchange (not for voice calls and SMS), i.e., what is commonly referred to as "mobile Internet" service). The probe is able to distinguish the transport protocol used for the communication (Transport Control Protocol, TCP, or User Datagram Protocol, UDP) and to categorize the traffic by application typology. All user identifiers and sensible information were irreversibly anonymized by Orange Labs before analysis. The probe collects data with 6-minute interval sessions, assigning the session to the cell identifier of the last used antenna. The data are recorded on a per user basis and cover more than 1,5 million of French mobile phone users in the Parisian metropolitan area, the "Ile-de-France", giving about 100 million records per day.

We limit the study in the paper to the "La Defense" region, a major business district in the northwest of Paris. The region is decomposed as shown in Fig. 2 at base station level, where red dots represent the base stations and the surrounding polygons represent the Voronoi cells. The size of a Voronoi cell depends on two basic factors: the geographical position and the coverage area (determined according to power level) of the corresponding base station.

We analyze the data in a normal working day from 8 am to $10 \mathrm{am}$ when people make their regular home-to-work travel. We choose this period to capture users mobility in the region. Upon this selection, we extract data consumption of about 20000 users.

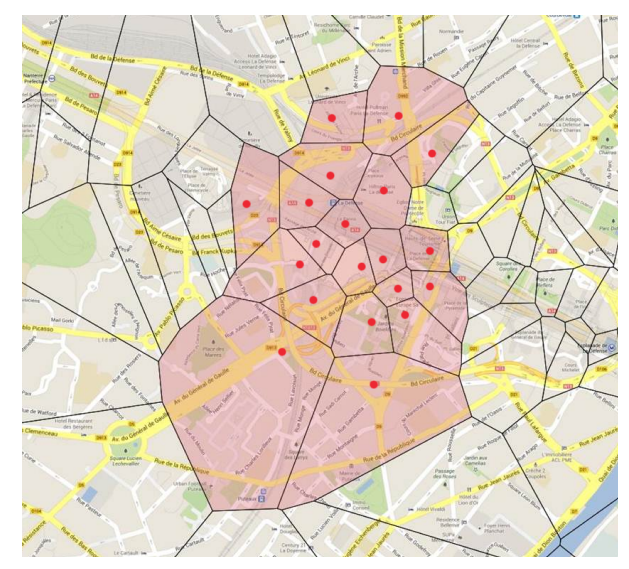

Figure 2: The dataset region. 


\subsection{Data Consumption Characteristics}

Before delving into the different offloading over Passpoint policies we define and analyze, we provide in this section some useful information about data consumption trends. First, we clearly highlight the most widely used applications and communication protocols. Then, we compare users consumption and demands.

Fig. 3 represents the proportion of traffic generated from each application (i.e., the traffic volume generated from each application to the total volume generated by all applications). We can clearly see that video streaming applications occupy the highest consumption portion (36\%) among other applications. These habitudes have taken place thanks to computing enhancements in mobile handheld devices and the increasing bandwidth from high-speed mobile networks in urban environments. This trend is also expected to increase at rapid paces in the coming years with the deployment of $4 \mathrm{G}$ networks. By classifying the data with respect to the transportlevel protocol only (i.e., TCP and UDP, used for applications needing or not, respectively, flow control and packet retransmissions upon loss, so roughly corresponding to non-interactive and interactive real-time services), we find out that TCP based applications are much more used than UDP ones (i.e., 97\% of the traffic is TCPbased whilst only $3 \%$ is UDP based). It is worth noting that video streaming applications are nowadays mostly based on HTTP Live Streaming protocol (also known as HLS) [22].

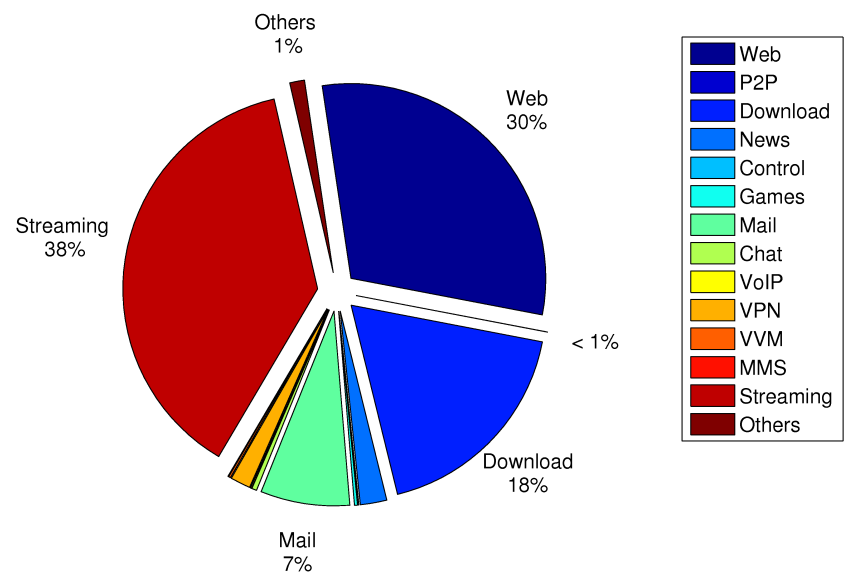

Figure 3: Traffic consumption by application type (3\% UDP, 97\% TCP; video streaming is mostly over TCP).

Comparing users' demands separately, Fig. 4 plots the user demand distribution given the 6-minute aggregation intervals (i.e., one cannot know through the data the instantaneous user demands because the collected data are aggregated as mentioned above). We can notice that while $97 \%$ of users have a very low demand of less than $1 \mathrm{MB}$ during the $6 \mathrm{~min}$ session (i.e., roughly $30 \mathrm{~kb} / \mathrm{s}$ on average), we have only $1 \%$ of them with a demand of more than 100 $\mathrm{MB}$ (i.e., roughly $3 \mathrm{Mb} / \mathrm{s}$ on average) and the maximum demand is about 325 Megabytes that corresponds to a mean bit rate of roughly $7.2 \mathrm{Mb} / \mathrm{s}$

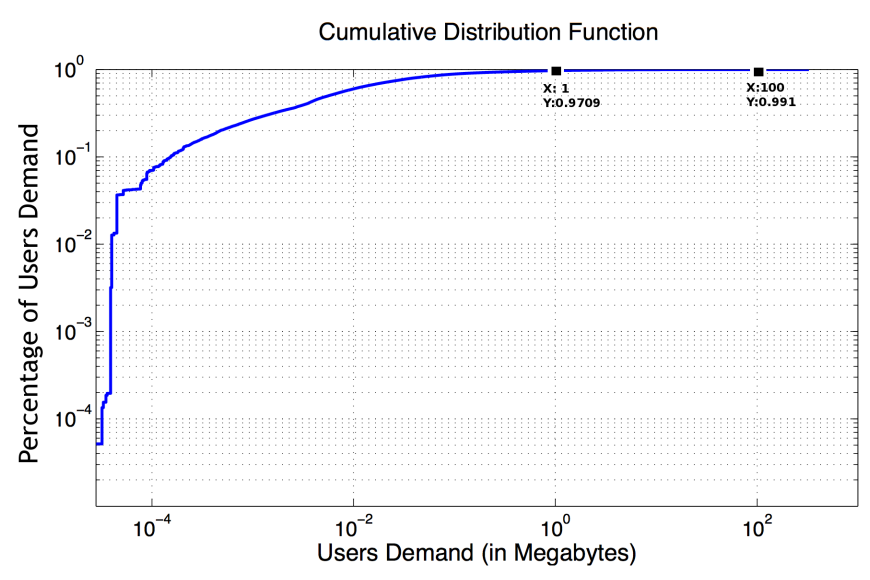

Figure 4: Cumulative Distribution Function of users demands.

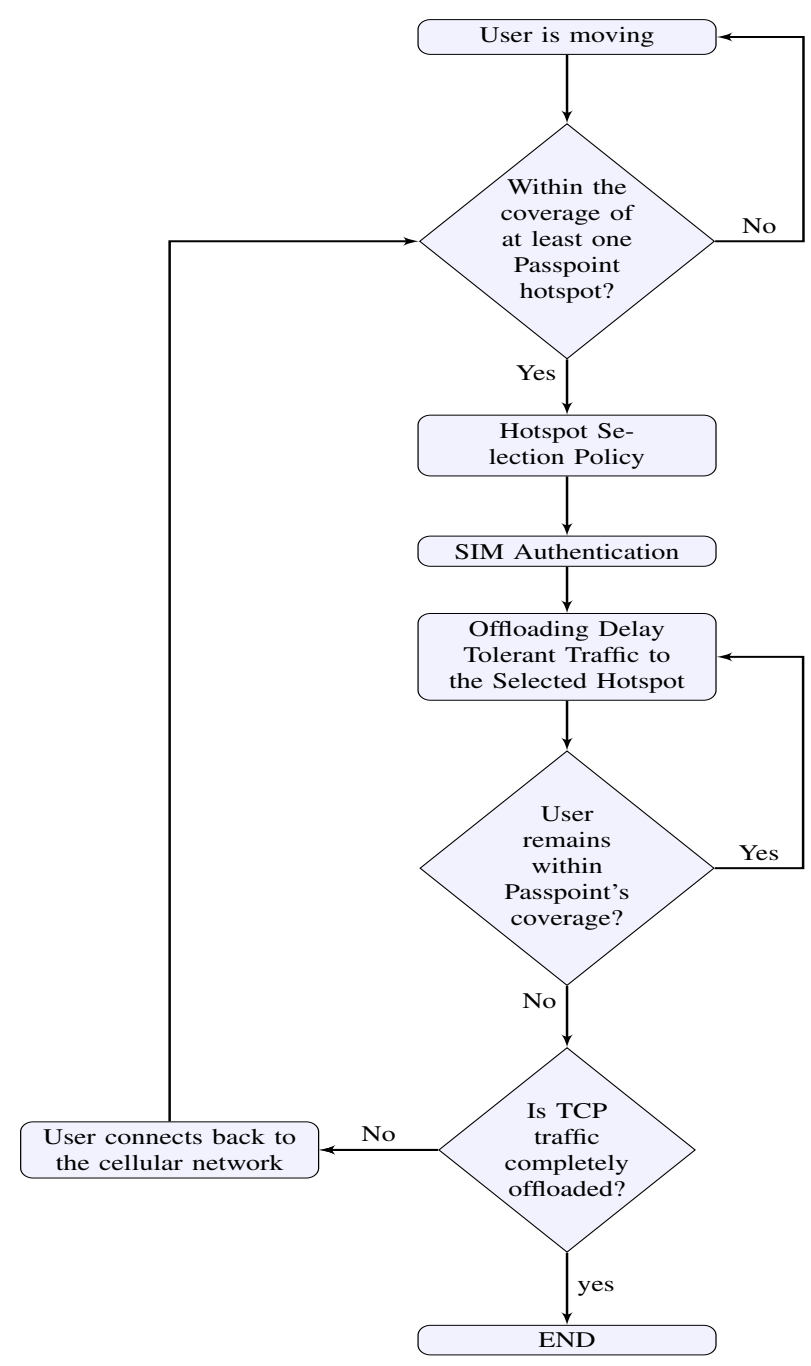

Figure 5: Offloading algorithm.

\section{EVALUATION METHODOLOGY OF OF- FLOADING OVER PASSPOINT}


In this section, we describe the methodology we adopted to evaluate mobile data traffic offloading over Passpoint hotspots. We draw the whole offloading procedure in the flow chart presented in Fig. 5.

Given a sample geographical distribution of Passpoint hotspots, we extract user displacement information from the data. Along its trajectory, a mobile device encountering a Passpoint hotspot, or a number of Passpoint hotspots at one location, can learn about the service providers available via each of them, as well as other characteristics of the hotspot, via the ANQP protocol. Thanks to this signaling, the mobile device can discover a comprehensive profile of the hotspot before association, so it can quickly identify, prioritize hotspots suitable for its needs and select the best match while still in the user's pocket.

The hotspot selection policy is therefore of paramount importance for both the user, able to associate to the best access point, and the network, which should avoid hotspot and backhauling link congestion. We compare in this paper three different hotspot selection policies, each taking into consideration one different parameter, as described in the following

1. Number of Associated Devices: the user is attached to the hotspot with the least number of associated devices (this information is provided by the hotspot in its response to the ANQP query as presented in Table 1).

2. Channel Utilization: the user is attached to the hotspot with the least Channel Utilization defined as the percentage of time the hotspot senses the medium busy (i.e., this information is also provided by the hotspot in its response to the ANQP query).

3. Signal Quality: the user is attached to the hotspot with the best received signal power.

While the first two are retrievable information via the ANQP Passpoint signaling, the latter instead does not strictly depend on Passpoint and can be considered as a policy that could easily be implemented with a relatively limited programming of mobile device's drivers ignoring hotspot capabilities.

After selecting the suitable hotspot, the mobile device is automatically authenticated. In Passpoint, this is done using Extensible Authentication Protocols (EAP) based on a Subscriber Identity Module (SIM) authentication, an authentication that is widely used in cellular networks today [5]. This procedure is specified in such a way that the process is completely transparent to the subscriber and that consistently works in any Passpoint network.

Then, the offloading process starts; only delay-tolerant traffic is offloaded to Passpoint hotspots, while retaining delay-sensitive traffic in mobile cellular networks. The delay-tolerant traffic is nothing else than TCP traffic. If the user moves out of the coverage of the Passpoint hotspot and finds no other hotspots in the environment, it returns back to the cellular network transparently.

\section{SIMULATION RESULTS}

In this section we describe the simulation framework we adopted to evaluate different offloading policies in MATLAB. For each simulation, the Passpoint hotspots are distributed in the selected region presented in Fig. 2 of approximately $1 \mathrm{~km}^{2}$. The results are obtained over many simulation instances, with a margin error lower than 3\%; we do not plot corresponding confidence intervals for the sake of presentation. In the following, we first present the radio model then we compare different offloading policies and hotspot placement strategies.

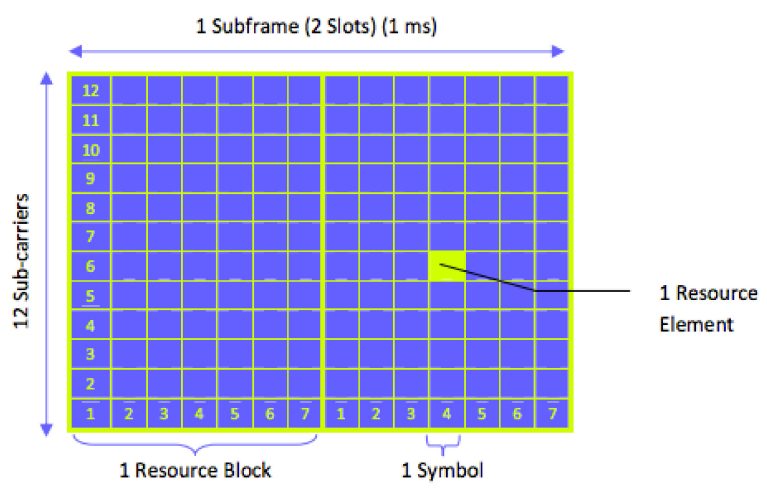

Figure 6: OFDMA frame structure.

\subsection{Radio Model}

The macrocells are assumed to operate using the OFDMA technology (e.g., in LTE) whose frame structure is based on time-frequency slots (see Fig. 6), also called tiles or resource blocks (RBs). A set of parameters for typical transmission bandwidths for LTE in the downlink is shown in Table 2, where the subcarrier spacing is $\Delta f=15 \mathrm{kHz}$. We select $20 \mathrm{MHz}$ as the transmission bandwidth, therefore the number of resource blocks per frame is equal to $100 \mathrm{RBs}$, e.g., allowing a max throughput of $100.8 \mathrm{Mb} / \mathrm{s}$ for the 64 QAM modulation.

These parameters are used to compute user demands in terms of RBs knowing only the volume in bytes. We note here that the modulation used by each user depends on its Signal to Noise plus interference (SINR) level and the path loss. We use the COST-231 Hata path loss model [23], devised as an extension to the Okumura-Hata model, which is the most widely used radio frequency propagation model for predicting the behavior of cellular transmissions in urban areas [24].

For the Passpoint hotspots, we employ a SINR interference model. Each hotspot is assigned one channel from the 13 available channels in France on the $2.4 \mathrm{GHz}$ frequency range. If the hotspot $j$ transmits signals to user $i$, the SINR computed by user $i$ is expressed as follows:

$$
\operatorname{SINR}_{i}=\frac{P d(i, j)^{-\alpha}}{N+\sum_{k \in A, k \neq j} P \lambda(i, k) d(i, k)^{-\alpha}}
$$

where:

$P$ is the transmission power of the hotspot (i.e., for simplicity we assume all hotspots use the same transmission power $\mathrm{P}$ of $20 \mathrm{dBm}$ ); $d(i, j)$ is the distance between user $i$ and the hotspot $j$;

$\alpha$ is the path loss index (a value typically between 2 and 4); $N$ is the background noise (i.e., we set this value to $-96 \mathrm{dBm}$ ); $A$ is the group of the hotspots existing in the network;

$\lambda(i, k)$ is the channel overlapping degree between the channels used by $i$ and $k$; it decreases when the channel distance between $i$ and $j$ increases. The channel overlapping degree is computed by [25] and shown in Table 3 . We note that when the channel distance is 5 or above, the overlapping degree becomes negligible. The maximum achievable capacity of access points is set to $54 \mathrm{Mbps}$ while the mean connection establishment time to a Passpoint hotspot is set to 12.9 seconds as in [26].

In the following, we compare various scenarios with respect to the capacity gain (CG) that we can get by offloading users traffic to Passpoint hotspots. The CG is defined as:

$$
C G=R B_{\text {freed }} / R B_{\text {total }}
$$


Table 2: Typical parameters for downlink transmission.

\begin{tabular}{|c||c|}
\hline Transmission bandwidth $[\mathrm{MHz}]$ & 20 \\
\hline Number of resource blocks & 100 \\
\hline OFDMA symbols per $1 \mathrm{~ms}$ & 14 \\
\hline Modulation symbol rate $(\mathrm{Mb} / \mathrm{s})$ & 16.8 \\
\hline QPSK Bit Rate $(\mathrm{Mb} / \mathrm{s})$ & 33.6 \\
\hline 16QAM Bit Rate $(\mathrm{Mb} / \mathrm{s})$ & 67.2 \\
\hline 64QAM Bit Rate $(\mathrm{Mb} / \mathrm{s})$ & 100.8 \\
\hline
\end{tabular}

where $R B_{\text {freed }}$ is the total number of $\mathrm{RBs}$ freed from the cellular mobile after offloading data traffic over Passpoint hotspots, and $R B_{\text {total }}$ is the total number of RBs required by users before offloading data traffic over Passpoint hotspots.

\subsection{Achievable gain with different hotspot se- lection policies}

Fig. 7 illustrates the capacity gain (in percentage) that we get for the three different selection policies with a random distribution of hotspots in the selected region. We can clearly notice that:

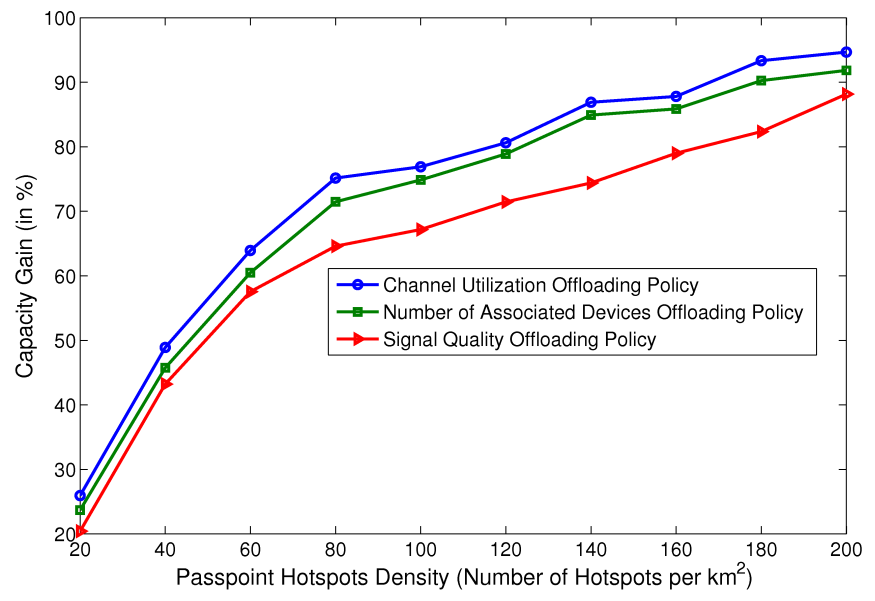

Figure 7: Capacity gain for different Passpoint hotspot selection policies.

- the capacity gain increases with the Passpoint density, as the probability of encountering a Passpoint while moving increases;

- the capacity gain with the Passpoint-agnostic Signal Quality policy gives results similar to those at the state of the art only for very high hotspot density, over 120 hotspot per square $\mathrm{km}$;

- the Channel Utilization offloading policy outperforms the other ones and offers the highest capacity gain. A reasonable justification of this behavior is that this policy equally distributes the users to hotspots taking into account traffic volume and hence allowing hotspot resources to be efficiently utilized;

- with the Signal Quality offloading policy, all users in a close location are assigned to the same hotspot because they will

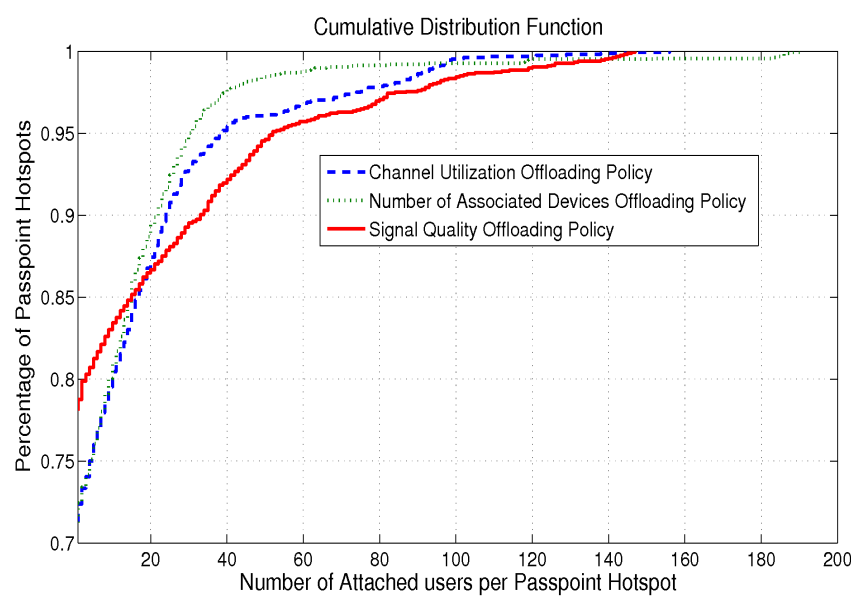

Figure 8: CDF of the number of attached users per Passpoint hotspot (density of 80 hotspots $/ \mathrm{km}^{2}$ ).

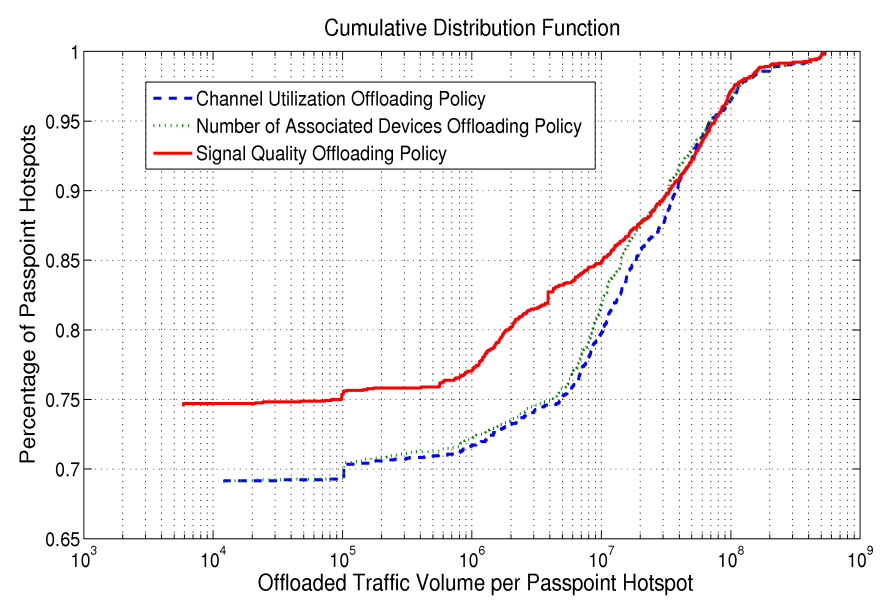

Figure 9: CDF of offloaded traffic volume per Passpoint hotspot (density of 80 hotspots $/ \mathrm{km}^{2}$ ).

all receive AP signals with the same power. As a result, there will be a larger number of users competing for limited resources in the unilaterally best hotspot whereas the resources in the other hotspots remain free and hence wasted;

- The Number of Associated Devices offloading policy does not take into account the traffic volume required by each user and thus inefficiently distributes the users to hotspots.

Furthermore, Fig. 8 and 9 show, respectively, the cumulative distribution function of the number of users attached as well as the traffic volume per Passpoint hotspot using the three offloading policies (for a hotspot density of 80 hotspots $/ \mathrm{km}^{2}$ ). We notice that the percentage of low-loaded hotspots is higher in the Signal Quality offloading policy than the other two policies. More precisely, with this Signal Quality offloading policy, approximately $80 \%$ of Passpoint hotspots have less than four attached users while $73 \%$ of hotspots have this value in the other two policies. Also $77 \%$ of hotspots offloading each less than $1 \mathrm{MB}$ of traffic in Signal Quality while $71 \%$ and $72.5 \%$ in Channel Utilization and Number of Associated Devices respectively. Moreover, the percentage of highlyloaded hotspots is bigger in Signal Quality offloading policy than 
Table 3: Channel overlapping degree.

\begin{tabular}{ccccccccr}
\hline Channel Distance & 0 & 1 & 2 & 3 & 4 & 5 & 6 & $\geq 7$ \\
\hline Overlapping Degree & 1 & 0.7272 & 0.2714 & 0.0375 & 0.0054 & 0.0008 & 0.0002 & 0 \\
\hline
\end{tabular}

the other two: $5 \%$ of hotspots with more than 52 users in Signal Quality while $3.5 \%$ in Channel Utilization and $1.5 \%$ for Number of associated devices; $11.5 \%$ of hotspots offloading each more than $30 \mathrm{MB}$ in Signal Quality and Number of Associated Devices policies to $13 \%$ in Channel Utilization offloading policy. These results confirm the previous findings and emphasize the more efficient usage of resources and distribution of traffic among different hotspots in the Channel Utilization offloading policy.

All in all, starting from a discrete Passpoint hotspot density, the gain of using the best among Passpoint offloading policies (i.e., the Channel Utilization one) and the offloading policy implementable without Passpoint (the Signal Quality one) is of roughly 15\%. These results are obtained for a random distribution of Passpoint hotspots, so the next question to answer is what is the most appropriate hotspot placement scheme.

\subsection{Passpoint placement schemes}

We compare different Passpoint placement schemes in order to assess the impact of Passpoint positions on the offloading system performance. Given the base station antenna-centric nature of cellular access, and more generally of wireless access, we consider different placement schemes depending on a parameter expressing the Distance To Borders (DTB) defined as:

$$
D T B_{i, j}=\frac{\operatorname{distance}\left(P_{i}, \diamond_{j}\right)}{\operatorname{distance}\left(M_{j}, \diamond_{j}\right)}
$$

where:

$P_{i}$ is the $i^{\text {th }}$ Passpoint and $M_{j}$ is the $j^{\text {th }}$ macrocell in the region. $\diamond_{j}$ is the polygon that surrounds the coverage area of Macrocell $j$. $\operatorname{distance}\left(P_{i}, \diamond_{j}\right)$ is the minimal distance from the Passpoint $P_{i}$ to all ribs of $\diamond_{j}$.

Based on the DTB parameter, we select four different placement schemes, presented in Fig. 10 where the colored zone represents the region of installing the Passpoint hotspots. We consider the placement of Passpoint hotspots in the:

- outer annulus (i.e., zone close to the edge) of the macrocell coverage, with a DTB between 0 and 0.33, as in Fig. 10(a);

- middle annulus (i.e., central zone) of the macrocell coverage, with a DTB between 0.33 and 0.66 , as in Fig. 10(b);

- inner annulus (i.e., zone closest to the base station) of the macrocell coverage, with a DTB between 0.66 and 1, as Fig. 10(c);

- whole macrocell zone, randomly distributed, with a DTB between 0 and 1, as in Fig. 10(d).

Fig. 11 illustrates the results obtained by varying the hotspot placement schemes. We consider here the Channel Utilization policy which appears as the best Passpoint offloading policy. We can clearly notice that:

- the hotspot placement with DTB between 0 and 0.33 (i.e., installing Passpoint hotspots in the outer annulus of the macrocell coverage) is the best placement scheme, which guarantees the highest capacity gain. The interpretation is straightforward as users located at the edge of the macrocell base

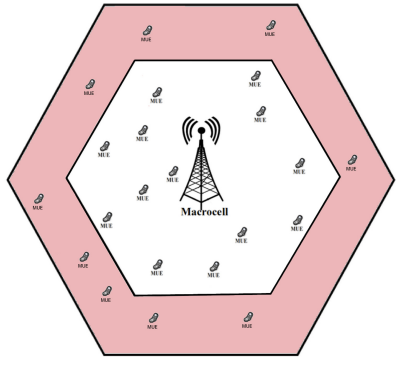

(a) DTB between 0 and 0.33

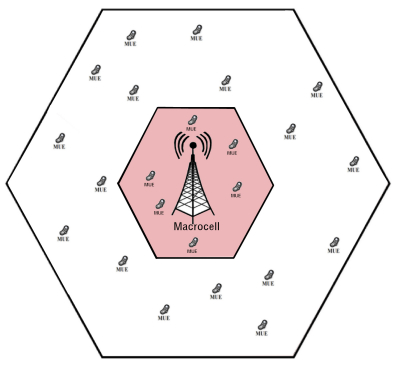

(c) DTB between 0.66 and 1

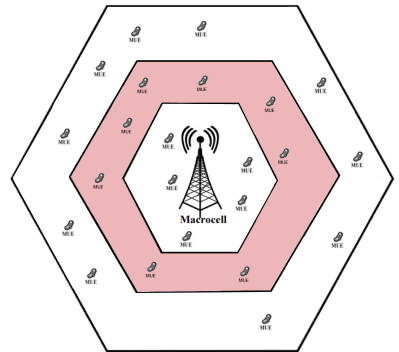

(b) DTB between 0.33 and 0.66

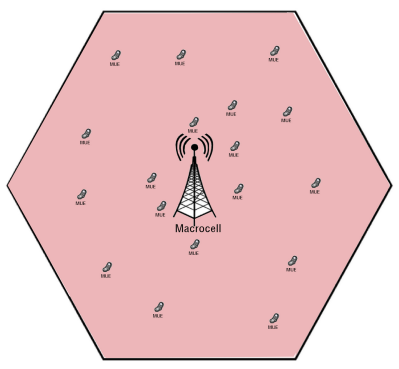

(d) DTB between 0 and 1
Figure 10: Illustration of different hotspot placement schemes.

station suffer from a low SINR; therefore, the modulation chosen for those users is the one that requires the least number of bits per symbol (i.e., QPSK modulation in our case) to reduce the symbol error rate. Those users have low bit rates and thus require more time and more RBs to transmit their traffic. By offloading their traffic to Passpoint hotspots, we free a big number of RBs from the cellular networks.

- The topology corresponding to DTB between 0.66 and 1 (i.e., inner annulus) is the worst among others. Differently than for the outer annulus case, users close to the macrocell base station use the modulation that requires the highest number of bits per symbol: those users have a high bit rate and require less time and RBs. So offloading their traffic is not very beneficial for cellular networks.

- The topology corresponding to DTB between 0 and 0.33 overcomes the random one (DTB between 0 and 1) with a mean capacity gain of roughly $5 \%$, and that with DTB between 0.33 to 0.66 (i.e., central annulus) with a mean gain of roughly $3 \%$.

As a final analysis, we are interested in comparing once more the different offloading policies under the best hotspot placement scheme, i.e., the case where Passpoint hotspots are placed only in the outer annulus. Fig. 12 illustrates the obtained results, where the dotted lines refer to the random hotspot placement replicated from Fig. 7. The figure shows that the gap between Passpoint policies and the signal quality policy is further increased when placing the hotspot in the outer annulus only. We notice a mean difference between the outer and random placement schemes of around $11 \%$ for 


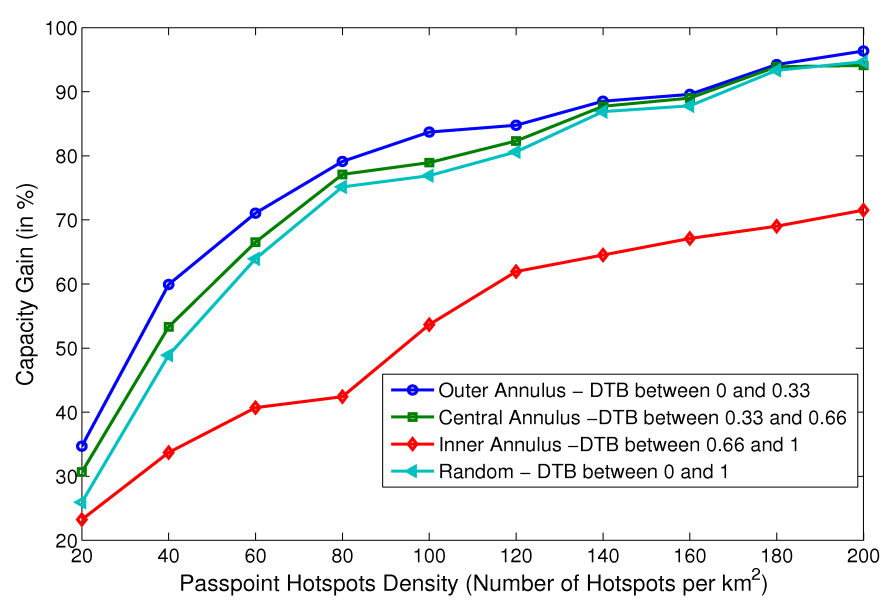

Figure 11: Capacity gain for different hotspot placement schemes under the best hotspot selection policy.

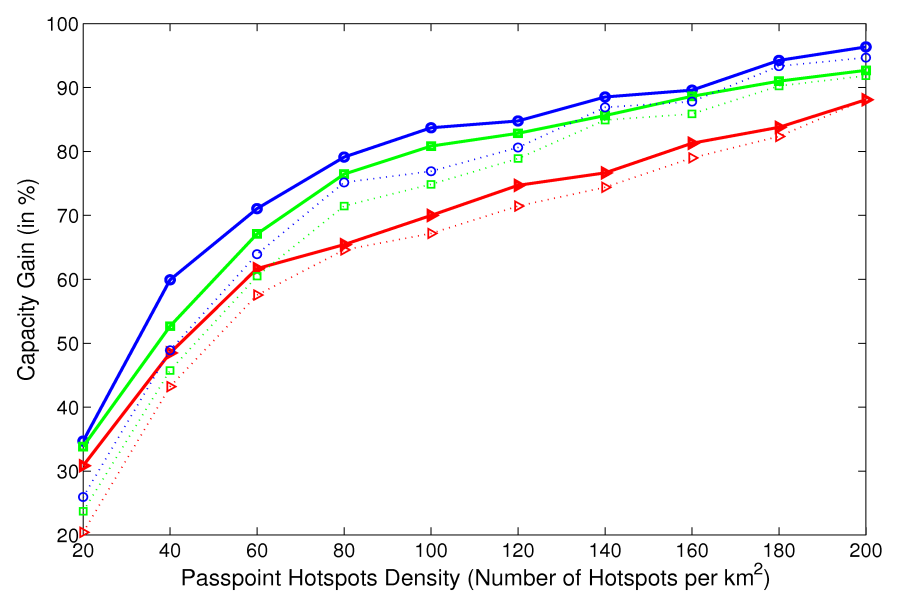

Figure 12: Capacity gain for different Passpoint hotspot selection policies under the best placement scheme.

low hotspots density and this difference decreases for high hotspots density with a mean difference of around 3\%. Overall, with hotspot placement in the outer annulus, the gain increases when using the Passpoint-enabled offloading policies rather than the signal quality one and this gain is around $15 \%$.

\section{CONCLUSION}

Traffic growth is outstripping the capacity of cellular mobile networks, especially in urban and densely populated zones. Moreover, operators are under pressure to find solutions to keep up with their customer's insatiable demand for data intensive applications. Data traffic offloading to Wi-Fi hotspots has always been an attractive solution for catering the increasing data demand in mobile networks, despite the existence of some drawbacks that limit their usage. Nowadays, with the advent of the Passpoint Certified Program [4], offloading data traffic to Passpoint hotspots is back to the forefront. The Passpoint Certified Program was created to address critical business needs for mobile data, streamline access and to help ease operator data traffic offload to these smart Wi-Fi networks in a completely transparent way for the user.

In this paper, we compare different conceivable mobile data traf- fic offloading over Passpoint hotspots to each other and to baseline approaches, using real mobile consumption data gathered from the Orange mobile network in Paris. First, we provide a brief analysis of mobile data consumption and characteristics. Then, we compute the capacity gain that one can get by offloading users traffic while taking into account different offloading policies and hotspot placement schemes. In particular, we show that offloading using Passpoint control-plane information can grant up to $15 \%$ gain with respect to Passpoint-agnostic ones based on signal quality information. As of our knowledge, our study is the first one quantifying the achievable cellular traffic offloading gain to Passpoint hotspots using the additional information given by Passpoint, via the ANQP protocol to mobile users, for hotspot selection; and this sort of offloading will surely play an important role in solving smart city challenges in the near future.

\section{Acknowledgment}

This work was partially supported by the ANR ABCD project (Grant No: ANR-13-INFR-005), and by the EU FP7 IRSES MobileCloud Project (Grant No. 612212).

\section{REFERENCES}

[1] Cisco, San Jose, CA, "Cisco Visual Networking Index: Global Mobile Data Traffic Forecast Update, 2012-2017, http://www.cisco.com/en/US/solutions/ collateral/ns341/ns525/ns537/ns705/ ns827/white_paper_c11-520862.pdf, Feb. 2013.

[2] D. Srinivasan, J. Dey, S. Kumar and R.N. Mukherjee, "Data Offload Approches for Mobile Operators: Improving Network Efficiency And Strengthening Quality Of Service, Wipro Technologies 2012.

[3] W. Lehr and L.W. Mcknight "Wireless Internet access: 3G vs. WiFi?", Telecommunications Policy, Vol. 27, pp. 351 370., 2003.

[4] "Wi-Fi Certified Passpoint Architecture for Public Access," White Paper, Aruba Networks, 2012.

[5] Wi-Fi Alliance, "Launch of Wi-Fi CERTIFIED Passpoint Enables a New Era in Service Provider Wi-Fi Austin, TX, June, 2012.

[6] A. Schumacher and J. Schlien, "WLAN Traffic Offload in LTE White Paper, November 2012.

[7] Femto forum, "Femtocell - Natural Solution for Offload", White paper, June, 2010.

[8] Femto forum, "Challenges in Deployment of UMTS/HSPA Femtocell", White paper, Feb, 2008.

[9] J.G. Andrews, H. Claussen, M. Dohler, S. Rangan, M.C Reed"Femtocells: Past, Present, and Future", IEEE Journal on Selected Areas in Communications , 2012.

[10] J. Roh , Y. Ji, Y.G. Lee, I. Rhee, T. Ahn "Femtocell Traffic Offload Scheme for Core Networks", 4th IFIP International Conference on New Technologies, Mobility and Security (NTMS), Paris, February 2011.

[11] D. Lopez-Perez, A. Ladanyi, A. Juttner, J. Zhang "OFDMA femtocells: Intracell Handover for Interference and Handover Mitigation in Two-Tier Networks", in Proc. of Wireless Communications and Networking Conference WCNC, April 2010.

[12] V. Chandrasekhar, J. Andrews, "Spectrum allocation in tiered cellular networks", IEEE Trans. Communications, Vol. 57, No. 10, pp. 3059- 3068, Oct. 2009. 
[13] S. Hoteit, S. Secci, R. Langar, G. Pujolle and R. Boutaba "Bankruptcy Game Approach for Resource Allocation in Cooperative Femtocell Networks", IEEE Global Communications Conference (IEEE GLOBECOM 2012), Anaheim, CA, USA, December 2012.

[14] A. Hatoum, N. Aitsaadi, R. Langar, R. Boutaba, and G. Pujolle, "FCRA: Femtocell Cluster-based Resource Allocation Scheme for OFDMA Networks", in Proc. of IEEE ICC 2011.

[15] K. Sundaresan, M. Arslan, S. Singh, S. Rangarajan, and S. Krishnamurthy, "FluidNet: a flexible cloud-based radio access network for small cells", in Proc. of the 19th annual international conference on Mobile computing and networking (MobiCom 2013), Miami, USA, October 2013.

[16] A. Balasubramanian, R. Mahajan, and A. Venkataramani "Augmenting mobile 3G using WIFI", in Proc. of ACM MOBISYS, 2010.

[17] K. Lee, J. Lee, Y. Yi, I. Rhee, S. Chong, "Mobile Data Offloading: How Much Can Wi-Fi Deliver?", IEEE/ACM Transactions on Networking, Vol. 21, No. 2, April 2013.

[18] A. Aijaz, O. Holland, P. Pangalos, H. Aghvami, "Energy savings for cellular access network through Wi-Fi offloading", IEEE International Conference on Communications, ICC, 2012.
[19] X. Hou, P. Deshpande, S.R. Das "Moving bits from 3G to metro-scale WiFi for vehicular network access: An integrated transport layer solution", in Proceedings of IEEE ICNP, Vancouver, Canada (2011)

[20] X. Zhuo, W. Gao, G. Cao, and Y. Dai, "Win-coupon: An incentive framework for $3 \mathrm{G}$ traffic offloading", in Proc. IEEE ICNP, 2011.

[21] D. Zhang, C.K. Yeo, "Optimal handing-back point in mobile data offloading," IEEE Vehicular Networking Conference (VNC), 2012.

[22] C. Mueller, S. Lederer and C. Timmerer "An Evaluation of Dynamic Adaptive Streaming over HTTP in Vehicular Environments," in Proc. of the 4th Workshop on Mobile Video (MoVid12), 2012.

[23] COST Action 231, "Digital mobile radio towards future generation systems, iňĄnal report," tech. rep., European Communities, EUR 18957, 1999

[24] M. Hata, "Empirical formula for propagation loss in land mobile radio services," IEEE Transactions on Vehicular Technology, vol. VT-29, pp. 317-325, 1981.

[25] M. Burton, "Channel overlap calculations for $802.11 \mathrm{~b}$ networks," White Paper, Cirond Technologies Inc., 2002.

[26] V. Bychkovsky, B. Hull, A. Miu, H. Balakrishnan and $S$. Madden "A measurement study of vehicular Internet access using in situ Wi-Fi networks," In Proc. of ACM MobiCom Conference, 2006. 